\title{
SCIDiCe
}

\author{
International Journal of Dentistry and Oral Science (IJDOS) \\ ISSN: 2377-8075
}

\section{The Efficacy of Hyaluronic Acid Gel in Pain Control of Recurrent Aphthous Stomatitis}

Research Article

Koray $\mathrm{M}^{1 *}$, Ofluoglu $\mathrm{D}^{1}$, Senemtasi $\mathrm{A}^{1}$, İssever $\mathrm{H}^{2}$, Yaltirik $\mathrm{M}^{1}$

${ }^{1}$ Istanbul University, Faculty of Dentistry, Department of Oral and Maxillofacial Surgery, Istanbul, Turkey.

${ }^{2}$ Istanbul University, Faculty of Istanbul Medicine, Department of Public Health, Istanbul, Turkey.

\section{Abstract}

This study was conducted to evaluate the efficacy of topical hyaluronic acid gel (HA) and compared with triamcinolone acetonide pomad (TA) in the treatment and pain control of RAS. A total of 57 patients with a history of RAS and currently suffering from oral ulcer were selected from patients referred to clinic. The subjects randomly divided into two groups; one group have received HA gel (Aftamed ${ }^{\mathbb{B}}$ Oral gel, AktiFarma. Istanbul, Turkey) the other group conceived TA pomade, (Kenacort-A Orabase ${ }^{\circledR}$ Pomade, 0,1\% Triamsinolon acetonide, Bristol-Myers Squibb Ilaclar Inc. Istanbul, Turkey). All patients were instructed to apply the agents to the ulcer 4 times per day for 7 days (day 0 to day 6). To evaluate pain level, a visual analog scale (VAS) was used. Although there was no statistical difference between two groups by means of mean ulcer VAS scores $(8.57 \pm 1.05$ in HA group and $8.59 \pm 1.08$ in TA group) at day 0 , significant differences were found at day 4 (VAS score $=4.88 \pm 0.83$ in HA group and 5.82 \pm 1.07 in TA group) and day 7 (VAS score $=2.30 \pm 0.90$ in HA group and $3.07 \pm 0.97$ in TA group). The ulcer pain score (VAS) of two groups decreased within time however, the pain score in HA group was statistically lower than that of the TA groups at day 4 and $7(\mathrm{p}<0.05)$. HA gel reduced the painfull symptoms of RAS when compared with TA pomad. These outcomes suggest that HA gel can be effectively used for pain control in RAS treatment.

Keywords: Hyaluronic Acid; Triamcinolone Acetonide; Recurrent Aphthous Stomatitis.

\section{Introduction}

Recurrent aphthous stomatitis (RAS) is a multifactorial chronic inflammatory disorder, characterized by recurrent, round or ovoid, painful ulcerations of the non-keratinized mucosa with a shallow necrotic center covered by a pseudomembrane and surrounded by an erythematous halo [1]. The current therapeutic approaches aim to relieve pain, alleviate inflammation, decrease functional disability, promote ulcer healing as well as reduction of the ulcer duration, frequency of recurrences and increase diseasefree period. Several topical medicaments including antibiotics, local analgesics, glucocorticoids, astringents, and laser therapy have been used for treatment $[1,2]$.

Triamcinolone acetonide (TA) is a fluoride synthetic corticosteroid and available in cream $(0.1 \%)$ and ointment $(0.1 \%)$ forms for topical use. The absorption rate varies from $1 \%$ to $36 \%$ in differ- ent parts of the body and increases via damaged, inflammed or dressed skin [3, 4]. Topical TA ointment was shown to be effective in the treatment of aphthous lesions [4, 5]. Hyaluronan or hyaluronic acid (HA) is a biomaterial that has been introduced as an alternative approach to enhance wound healing [6]. HA is a major carbohydrate component of the extracellular matrix and can be found in many tissues [7]. In the treatment of RAS, hyaluronic acid rapidly reduce the pain and discomfort caused by the ulcers, accelerated the healing process, and significantly reduce the risk of recurrence of the disorder. It also controls the inflammatory process and rehydrates the tissues [8]. This study was conducted to evaluate the efficacy of topical HA gel and compared with TA pomade in pain control of RAS.

\section{Materials and Method}

Sixty patients (mean age $=38.16 \pm 12.2$; range $=18-60$, female $=30$,

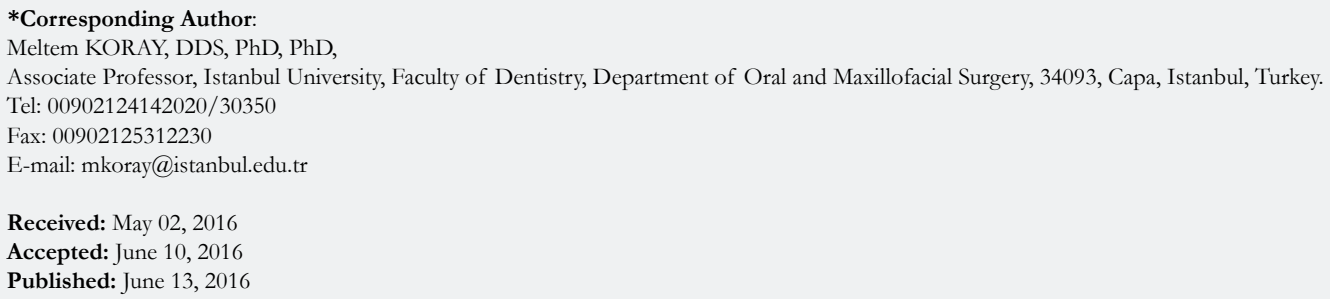

Copyright: Koray $\mathbf{M}^{\circ}$ 2016. This is an open-access article distributed under the terms of the Creative Commons Attribution License, which permits unrestricted use, distribution and reproduction in any medium, provided the original author and source are credited. 
male $=30$ ) whom were referred to the Department of Oral and Maxillofacial Surgery with the complaint of oral mucosal ulcers were included in the study. Mean disease time was $8.28 \pm 4.9$ year and mean healing time was $9.80 \pm 2.8$ day in all patients. All of the participiants received written and verbal information about the study and signed a detailed informed-consent form voluntarily. The subjects randomly divided into two groups; TA pomade or HA gel. The diagnosis of RAS was based on the anamnesis and clinical examination [9]. Participants fullfilled the following inclusion criterias; being over the age of 18 years; having an history of RAS for at least 2 years, having only one well-demarcated ulcer in easily accessible area of the mouth for less than 48 hours' duration and having normal sense of pain without anesthesia or paresthesia.

The exclusion criterias were as follows; pregnancy and lactation; having a hematological deficiency such as anemia, iron, vitamin B12 and/or folic acid deficiency that could pose a risk for RAS; systemic diseases such as ulcerative colitis, Crohn's disease, Behçet's syndrome alcohol and smoking consumption; treatment of ulcers with systemic steroids, vitamines, antibiotics, antihistamines, oral retinoids or immunomodulatory agents within three months before study entry.

The sociodemographic datas and clinical characteristics of the ulcer cases were collected by questionnaire regarding age, gender, the mean disease duration, the mean healing time of previous ulcers, family RAS history, and the localization of the current ulcer. To evaluate pain level, a visual analog scale (VAS) consisting of a $10-\mathrm{cm}$ horizontal line between the poles of "no pain $(0)$ " to "unbearable pain (10)" was used [10]. After clinical examinations and measurement of baseline datas, patients were randomly asigned into one of two groups; TA group (Kenacort-A Orabase ${ }^{\circledR}$ Pomad, 0,1\% Triamsinolon acetonide, Bristol-Myers Squibb Ilaclar Inc. Istanbul, Turkey) or HA group (Aftamed ${ }^{\mathbb{R}}$ Oral gel, AktiFarma. Istanbul, Turkey). All patients were instructed to apply the agents to the ulcer 4 times per day (after meals and before bed time) for 7 days (day 0 to day 6). Ulcer pain level was measured at day 0, 4 and 7 and patients were warned not to use any other products for the treatment of aphthous ulcers while participating in this study. At the end of therapy, all patients were also asked to self-report any adverse effects of agents.

Analysis was performed using SPSS (Statistical Package for Social Sciences) for Windows 15.0. All variables were analyzed descriptively. For descriptive statistical methods (mean, Standard deviation and frequency), as well as for the comparison of quantitative data, a one-way ANOVA were performed for between-group comparisons of parameters showing a normal distribution. For the comparison of continuous variables with normal distributions, Student's $t$-test was performed.

\section{Results}

A total of 60 patients were enrolled the study and 3 patients dropped out because of violation of the study protocol and 28 subjects in TA group, 29 subjects in HA group completed the study. Statistically significant differences were not detected among the demographics and ulcer histories including age, gender, disease time, mean healing time, family RAS history and ulcer localization between two groups ( $p>0.05)$. Although there was no statistical difference between two groups by means of mean ulcer VAS scores $(8.59 \pm 1.08$ in TA group and $8.57 \pm 1.05$ in HA group) at day 0 , significant differences were found at day 4 (VAS score $=5.82 \pm 1.07$ in TA group and $4.88 \pm 0.83$ in HA group) and day 7 (VAS score $=3.07 \pm 0.97$ in TA group and $2.30 \pm 0.90$ in HA group). The ulcer pain scores (VAS) of two groups decreased within time however, the pain score in HA group was statistically lower than that of the TA group at day 4 and $7(\mathrm{p}<0.05)$ (Table 1$)$. All patients tolerated the agents and no side effect was reported during the study.

\section{Discussion}

RAS is a complicated condition and the precise etiology still remains unknown. Different treatment modalities, topical and systemic agents have been used to decrease symptoms, reduce ulcer number and size, increase disease-free periods [11]. The first line treatment of RAS should always start with topical medications. TA is a medium to high potency corticosteroid, a fluorinated prednisolone derivative and is being widly used in the treatment of mild to severe RAS [12-14]. Pain reduction is a recognized feature of steroid treatment for apthous ulceration. Rhodus and Bereuter found that $20 \%$ of patients treated with TA reported pain reduction in the first 3 days of treatment [15]. In another study Al-na'mah et al. compared a paste containing dexamethasone and TA in orabase in RAS treatment and reported faster healing of the ulcer with dexamethasone treatment although statistically not significant [16]. Deshmukh and Bagewadi have compared the efficacy of curcumin, which is known for its strong antioxidant, antiseptic, antibacterial, anti-inflammatory, immunomodulatory and analgesic properties, and TA in the gel form in the treatment of RAS and found similar reduction in size and number of ulcer in both groups [17]. Bhalang et al. reported a higher effectiveness of $0.1 \%$ TA than acemannan, a polysaccharide extracted from Aloe vera, in the treatment of oral aphthous ulceration [18].

$\mathrm{HA}$ is also an alternative agent for topical treatment in RAS. It is a glycosaminoglycan with anti-inflammatory and antiedematous effects. HA has multifaceted roles in biology, utilizing both its physicochemical and biological properties, and also has many properties that make it a potentially ideal molecule for assisting wound healing, inducing beneficial early granulation tissue forma-

Table 1. The comparison of triamcinolone acetonide (TA) group and hyaluronic acid (HA) group in regards of VAS score.

\begin{tabular}{|c|c|c|c|c|}
\hline VAS score & TA group $(\mathbf{n}=\mathbf{2 8})$ & HA group $(\mathbf{n}=\mathbf{2 9})$ & $\boldsymbol{t}$ & $\mathbf{p}$ \\
\hline Day of 0 & $8.59 \pm 1.08$ & $8.57 \pm 1.05$ & 0.072 & 0.9 \\
\hline Day of 4 & $5.82 \pm 1.07$ & $4.88 \pm 0.83$ & 3.37 & $0.001^{*}$ \\
\hline Day of 7 & $3.07 \pm 0.97$ & $2.30 \pm 0.90$ & 2.97 & $0.004^{*}$ \\
\hline
\end{tabular}

Student's $t$-test was used ${ }^{*} \mathrm{p}<0.05$ 
tion, inhibiting destructive inflammation during the healing phase, promoting re-epithelialization and also angiogenesis [19-21]. The use of HA has been established in many medical disciplines, such as ophthalmology, dermatology, and rheumatology [19, 22]. The topical application of $0.2 \% \mathrm{HA}$ gel seems to be an effective and safe therapy especially for reducing the number, healing period, pain, and area of ulcers, in patients with RAS [8] and it also immediately reduced discomfort and ulcer duration [23]. In a prospective double-blind, randomized clinical trial we have reported beneficial effects of HA spray in the management of swelling and trismus during immediate postoperative period following impacted third molar surgery [21].

Aftamed $^{\circledR}$ (high-molecular-weight hyaluronic acid 240mg/100g) gel contains polycarbophil, a protein that attaches to wet mucosa and this attachment provides direct mechanical protection and keeps the HA in place on the wound. It plays an integral role in maintaining and regulating the moisture level in tissues and facilitates the transport of nutrients into cells and the removal of metabolic waste. Al-Bayaty et al. evaluated the wound healing activities of Aftamed ${ }^{\circledR}$ and chlorine dioxide gels in streptozocin-induced diabetic rats. They reported that Aftamed ${ }^{\mathbb{B}}$-treated wounds exhibited significant increases in hydroxyproline, cellular proliferation, the number of blood vessels and the level of collagen synthesis so they concluded that Aftamed ${ }^{\circledR}$ gel is able to significantly accelerate the process of wound healing in diabetic rats [24].

The results of the study demonstrated that HA gel reduced the pain of RAS patients without any systemic or local side effects. Furthermore it was easy to apply without any unfavorable taste. In this study, patients treated with HA gel showed a significant improvement in pain intensity at days 4 and 7 when compared with TA group. These results showed the efficacy of HA gel in pain reduction. Although we did not evaluate the changes in ulcer size and confirm the effects of HA gel in size reduction of ulcer statistically, our experiences and patients feedbacks showed that HA gel is also effective in ulcer size reduction and enhanced healing. Topically adminestered Aftamed ${ }^{\circledR}$ gel exerts its beneficial effects on wound healing by stimulating the deposition of collagen and angiogenesis [25]. Angiogenesis increases the delivery of oxygen and other nutrients that are necessary for local collagen synthesis which plays a central role in the healing of wounds. In further studies involving larger number sample size and evaluating the effect of HA gel in cellular base might clarify the whole mechanism and also the efficacy of HA gel on RAS recurrence.

\section{Conclusion}

The results of this study demonstrate that topical application of HA gel could decrease pain intensity, without any side effects and easy appliable. Therefore HA gel could be used a well-tolerated, safe, topical therapeutic agent in clinical practice of RAS treatment.

\section{Acknowledgement}

The present study was supported by the Research Fund of Istanbul University. Project No. BEK-2016-22127.

\section{References}

[1]. Ship JA (1996) Recurrent aphthous stomatitis. An update. Oral Surg Oral Med Oral Pathol Oral Radiol Endod 81(2): 141-147.

[2]. Jurge S, Kuffer R, Scully C, Porter SR (2006) Mucosal disease series. Number VI. Recurrent aphthous stomatitis. Oral Dis 12(1): 1-21.

[3]. Khandwala A, Van Inwegen RG, Charney MR, Alfano MC (1997) 5\% amlexanox oral paste, a new treatment for recurrent minor aphthous ulcers: II. Pharmacokinetics and demonstration of clinical safety. Oral Surg Oral Med Oral Pathol Oral Radiol Endod 83(2): 231-238.

[4]. Fani MM, Ebrahimi H, Pourshahidi S, Aflaki E, Shafiee Sarvestani S (2012) Comparing the Effect of Phenytoin Syrup and Triamcinolone Acetonide Ointment on Aphthous Ulcers in Patients with Behcet's Syndrome. Iran Red Crescent Med J 14(2): 75-78.

[5]. Miles DA, Bricker SL, Razmus TF, Potter RH (1993) Triamcinolone acetonide versus chlorhexidine for treatment of recurrent stomatitis. Oral Surg Oral Med Oral Pathol 75(3): 397-402.

[6]. Hammad HM, Hammad MM, Abdelhadi IN, Khalifeh MS (2011) Effects of topically applied agents on intra-oral wound healing in a rat model: a clinical and histomorphometric study. Int J Dent Hyg 9(1): 9-16.

[7]. Chen WY, Abatangelo G (1999) Functions of hyaluronan in wound repair. Wound Repair Regen 7(2): 79-89.

[8]. Lee JH, Jung JY, Bang D (2008) The efficacy of topical $0.2 \%$ hyaluronic acid gel on recurrent oral ulcers: comparison between recurrent aphthous ulcers and the oral ulcers of Behçet's disease. J Eur Acad Dermatol Venereol 22(5): 590-595.

[9]. Porter S, Scully C (2005) Aphthous ulcers (recurrent). Clin Evid 13: $1687-$ 1694.

[10]. Liu J, Zeng X, Chen Q, et al. (2006) An evaluation on the efficacy and safety of amlexanox oral adhesive tablets in the treatment of recurrent minor aphthous ulceration in a Chinese cohort: a randomized, double-blind, vehicle-controlled, unparallel multicenter clinical trial. Oral Surg Oral Med Oral Pathol Oral Radiol Endod 102(4): 475-481.

[11]. Scully C, Gorsky M, Lozada-Nur F (2003) The diagnosis and management of recurrent aphthous stomatitis: a consensus approach. J Am Dent Assoc 134(2): 200-207.

[12]. Quijano D, Rodríguez M (2008) Topical corticosteroids in recurrent aphthous stomatitis. Systematic review. Acta Otorrinolaringol Esp 59(6): 298307.

[13]. Scully C, Porter S (2008) Oral mucosal disease: recurrent aphthous stomatitis. Br J Oral Maxillofac Surg 46(3): 198-206.

[14]. Elad S, Epstein JB, von Bültzingslöwen I, Drucker S, Tzach R, Yarom N (2011) Topical immunomodulators for management of oral mucosal conditions, a systematic review; Part II: miscellaneous agents. Expert Opin Emerg Drugs 16(1): 183-202.

[15]. Rhodus NL, Bereuter J (1998) An evaluation of a chemical cautery agent and an anti-inflammatory ointment for the treatment of recurrent aphthous stomatitis: a pilot study. Quintessence Int 29(12): 769-773.

[16]. Al-Na'mah ZM Carson R, Thanoon IA (2009) Dexamucobase: a novel treatment for oral aphthous ulceration. Quintessence Int 40(5): 399-404.

[17]. Deshmukh RA, Bagewadi AS (2014) Comparison of effectiveness of curcumin with triamcinolone acetonide in the gel form in treatment of minor recurrent aphthous stomatitis: A randomized clinical trial. Int J Pharm Investig 4(3): 138-141.

[18]. Bhalang K, Thunyakitpisal P, Rungsirisatean N (2013) Acemannan, a polysaccharide extracted from Aloe vera, is effective in the treatment of oral aphthous ulceration. J Altern Complement Med 19(5): 429-434.

[19]. Moseley R, Waddington RJ, Embery G (2002) Hyaluronan and its potential role in peri-odontal healing. Dent Update 29(3): 144-148.

[20]. Gontiya G, Galgali SR (2012) Effect of hyaluronan on periodontitis: a clinical and histological study. J Indian Soc Periodontol 16(2): 184-192.

[21]. Koray M, Ofluoglu D, Onal EA, Ozgul M, Ersev H, Yaltirik M, et al., (2014) Efficacy of hyaluronic acid spray on swelling, pain, and trismus after surgical extraction of impacted mandibular third molars. Int. J. Oral Maxillofac. Surg 43(11): 1399-1403.

[22]. Laurent TC, Fraser JR (1992) Hyaluronan. FASEB J 6(7): 2397-2404.

[23]. Nolan A, Baillie C, Badminton J, Rudralingham M, Seymour RA (2006) The efficacy of topical hyaluronic acid in the management of recurrent aphthous ulceration. J Oral Pathol Med 35(8): 461-465.

[24]. Al-Bayaty F, Abdulla MA (2012) A comparison of wound healing rate following treatment with aftamed and chlorine dioxide gels in streptozotocininduced diabetic rats. Evid Based Complement Alternat Med 2012: 468764.

[25]. Buemi M, Galeano M, Sturiale A, Ientile R, Crisafulli C, Parisi A, et al., (2004) Recombinant human erythropoietin stimulates angiogenesis and healing of ischemic skin wounds. Shock 22(2): 169-173. 